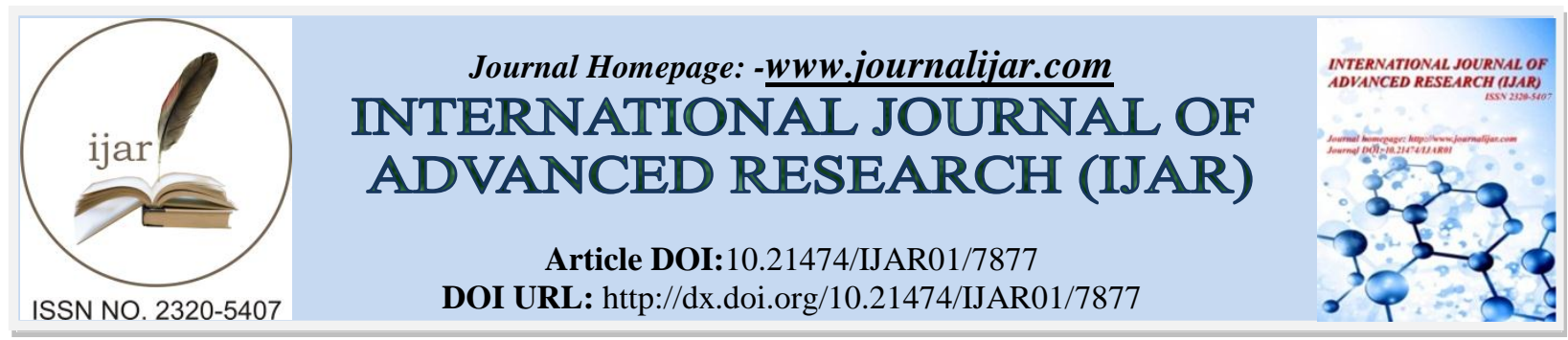

RESEARCH ARTICLE

\title{
LES ESPECES VEGETALES INDICATRICES DE LA FERTILITE DE SOLS DANS LA COMMUNE URBAINE D'AGUIE AU NIGER.
}

\author{
Abarchi Idrissa ${ }^{1}$, Seyni Boureima ${ }^{1}$, Karim Saley $^{2}$ and China Boureima ${ }^{2}$. \\ 1. Faculté d'Agronomie et des Sciences de l'Environnement, Université Dan Dicko Dankoulodo de Maradi, BP: \\ 465 Maradi, Niger. \\ 2. Faculté des Sciences et Techniques, Université Dan Dicko Dankoulodo de Maradi, BP: 465 Maradi, Niger.
}

\section{Manuscript Info}

\section{Manuscript History}

Received: 10 August 2018

Final Accepted: 12 September 2018

Published: October 2018

\section{Keywords:-}

phytosociological surveys, plant species, indigenous knowledge, soil fertility, Aguie.

\begin{abstract}
A study based on farmers' surveys and phytosociological surveys in the fields were conducted in four villages of the urban commune of Aguie to determine plant species indicators of soil fertility. In each of the four villages (Doramaoua, Dan Kada, Kodaou and Dan Tchiro), fifteen farmers were randomly surveyed. Results indicated that some plants species gives information on the soils status in which they were grown. Hence plants species indicators of good soil fertility are Piliostigma reticulatum, Faidherbia albida, Guiera senegalensis, Dichrostachys cinerea, Bauhinia rufescens, Prosopis africana, Acacia nilotica, Albizia chevalieri et Ziziphus mauritiana for trees; Merremia tridentata, Eragrostis tremula, Dactylotenium aegyptium, Commelina forkalaei, Cassia mimosoides, Sporobolus spicatus, Cenchrus biflorus et Corchorus tridens for herbaceous. However, plant species, such as: Striga hermonthica, Merremia tridentata and Eragrostis tremula are indicators of poor soils. Therefore, there is an indigenous knowledge based on plant species that allows to know the state of fertility of soils.
\end{abstract}

Copy Right, IJAR, 2018,. All rights reserved.

\section{Introduction:-}

Depuis que l'homme sait substituer à la végétation naturelle une autre végétation, cultivée dans le but de satisfaire ses besoins, il y a eu rupture de l'équilibre entre le sol, la végétation et le climat, exposant le sol aux aléas climatiques (Mathurin, 2007). Ce déséquilibre fait que le sol change non seulement de couleur, mais aussi de texture, de structure, d'épaisseur et de richesse en matière organique indispensable à la vie des plantes.

L'élément qui influence le plus directement le potentiel de production agricole d'un pays est la disponibilité de terres arables. La terre constitue en effet la ressource sans laquelle il ne peut y avoir de productions agro-sylvo-pastorales (FAO, 2001).

Aujourd'hui, aucun développement agricole ne peut être envisagé sans la prise en compte de la capacité des sols à produire des bons rendements. La fertilité des sols, est conditionnée par un ensemble de facteurs naturels ou artificiels qui interagissent pour permettre la pleine expression du potentiel de production. Ainsi, qu'elles soient physiques, chimiques ou biologiques, les différentes composantes de sols nécessitent d'être constamment entretenues et améliorées (CTIFL, 2012). 
Dans les années antérieures, les accroissements de production agricoles obtenus sont dûs à l'augmentation des surfaces cultivées et non à l'accroissement des rendements. Or aujourd'hui les surfaces cultivables sont saturées et on ne peut plus augmenter la production par accroissement des surfaces. Il se trouve que pour satisfaire les besoins croissants des populations, chaque champ doit produire davantage (Ali, 1997).

L'agriculture représente la principale activité de plus de $80 \%$ de la population active du Niger. Elle est pratiquée sur moins de $12 \%$ du territoire nationale et reste largement tributaire des aléas climatiques (INS, 2013). A cela s'ajoute la pauvreté des sols en matière organique et surtout en phosphore organique (Bationo et al., 1996). Cependant, depuis très longtemps, des savoirs indigènes permettant de sonder l'état de fertilité de sols. Ces savoirs locaux sont basés sur l'observation de l'état de surface du sol et les plantes qui y poussent entre autres. Si une plante pousse bien et sans infestation, son rendement sera satisfaisant. Et si ce rendement est obtenu sans utilisation des produits agrochimiques, on peut affirmer que le sol est fertile (Weller, 2013). En effet une plante ne pousse jamais au hasard, des qu'elle apparaisse, elle a un rôle à jouer dans cet endroit, à ce moment precis (Christel, 2006).

Cette étude a pour but de déterminer les espèces végétales indicatrices de fertilité de sols.

\section{Matériels et Méthodes:-}

\section{Site expérimental:-}

L'étude a été menée au niveau de quatre villages (Doramaoua, Dan kada, Kodaou et Dan Tchiro), situés respectivement à l'est, à l'ouest, au sud et au nord dans la commune d'Aguié (Tableau 1).

Tableau 1:- Coordonnées géographiques des villages

\begin{tabular}{|l|l|l|l|}
\hline \multirow{2}{*}{ Localités } & \multicolumn{1}{|c|}{ Coordonnées géographiques } & \multicolumn{1}{c|}{$\begin{array}{l}\text { Localisation par rapport } \\
\text { à la ville d'Aguié }\end{array}$} \\
\cline { 2 - 3 } & Latitude Nord & Longitude Est & EST \\
\hline Doramaoua & $13^{\circ} 29^{\prime} 52.2$ & $7^{\circ} 50^{\prime} 20.7$ & OUEST \\
\hline Dan kada & $13^{\circ} 30^{\prime} 50.5$ & $7^{\circ} 39^{\prime} 34.2$ & SUD \\
\hline Kodaou & $1^{\circ} 28^{\prime} 08.1$ & $7^{\circ} 46^{\prime} 01.4$ & NORD \\
\hline Dan Tchiro & $7^{\circ} 43^{\prime} 51.9$ & CENTRE \\
\hline Aguié & $13^{\circ} 36^{\prime} 30.5$ & $7^{\circ} 47^{\prime} 00.6$ & \\
\hline
\end{tabular}

Enquête ethnobotanique et Relevé phytosociologique:-

Dans chacun de ces villages, quinze paysans ont été enquetés de manière aleatoire.

Pour le relevé phytosociologique, au niveau de chacun de ces quatre terroirs, deux placettes de $2500 \mathrm{~m}^{2}(50$ $\mathrm{m} \times 50 \mathrm{~m})$ sont délimitées au niveau des champs à fertilité élevée (CFE), et au niveau des champs à faible fertilité (CFF), sur la base de l'état des cultures et des récoltes.

Le relevé des espèces non ligneuses s'est fait par la méthode des points quadrants (relevé linéaire). Le principe de cette méthode consiste à effectuer le long d'une ligne de $10 \mathrm{~m}$, le recouvrement par points espacés de $10 \mathrm{~cm}$. Les espèces qui sont touchées par l'aiguille sont notées sur la fiche de relevé linéaire. Un relevé linéaire fournit les informations sur les fréquences et contribution spécifiques des espèces.

La fréquence spécifique (ou fréquence centésimale) est définie comme étant le rapport du nombre (ni) de fois où l'espèce (i) a été recensée le long de la ligne au nombre totale $(\mathrm{N})$ de points échantillonnés.

$$
\text { FSi }(\%)=\frac{n i}{N} * 100
$$

La comparaison entre les fréquences spécifiques des espèces dans les CFE et celle dans les CFF permet ainsi de voir le niveau d'indicateur de fertilité ou de pauvreté du sol. Quant à la contribution spécifique (CSi), elle représente le rapport de la fréquence spécifique d'une espèce (i) à la somme des fréquences spécifiques de toutes les espèces recensées.

$$
\operatorname{CSi}(\%)=\frac{F S i}{\Sigma F S} * 100
$$




\section{Analyse des données:-}

Toutes les données collectées ont été saisies avec le tableur Excel. Ce logiciel a permis de calculer les différentes fréquences des espèces.

\section{Résultats:-}

\section{Enquête ethnobotanique:-}

Vingt quatre (24) herbacés et vingt deux (22) ligneux ont été évoqués par les exploitants tantôt en tant qu'indicateur de fertilité, tantôt en tant qu'indicateur de pauvreté du sol.

\section{Espèces ligneuses indicatrices de fertilité de sols:-}

Les espèces ligneuses indicatrices de la fertilité de sols et de la pauvreté des sols sont présentées au niveau du Tableau 2. Certaines espèces ligneuses comme Faidherbia albida (Del.) A. Chev., Bauhinia rufescens Lam., Prosopis africana (Guill . et Perr.) Taub., et Ziziphus mauritiana Lam. n'ont été citées qu'en tant qu'espèces indicatrices de fertilité de sols. Par contre Acacia nilotica (L) WILLD et Albizia chevalieri Harms sont répertoriées comme espèces indicatrices de la pauvreté de sols.

Tableau 2:-Ligneux indicateurs de fertilité de sols et de la pauvreté de sols

\begin{tabular}{|l|l|l|}
\hline Noms scientifiques & $\begin{array}{l}\text { Exploitants ayant considéré } \\
\text { l'espèce comme indicatrice de } \\
\text { fertilité de sols (\%) }\end{array}$ & $\begin{array}{l}\text { Exploitants ayant considérée } \\
\text { l'espèce comme indicatrice } \\
\text { de pauvreté de sols (\%) }\end{array}$ \\
\hline Acacia nilotica (L) WILLD. & 11,66 & 16,66 \\
\hline Acacia senegal (L) Willd. & 0 & 10 \\
\hline Albizzia chevalieri Harms & 5 & 16,66 \\
\hline Annona senegalensis Pers. & 10 & 1,66 \\
\hline Azadirachta indica A. Juss. & 0 & 5 \\
\hline Balanites aegyptiaca (L.) Del. & 8,33 & 3,33 \\
\hline Bauhinia rufescens Lam. & 20 & 0 \\
\hline Bombax costatum Pellgr. Et Vuillet & 0 & 5 \\
\hline Boscia senegalensis (Pers.) Lam. ex Poir. & 5 & 0 \\
\hline Combretum micranthum G. Don. & 6,66 & 3,33 \\
\hline Dichrostachys cinerea (L.) Wight et Arn. & 21,66 & 3,33 \\
\hline Faidherbia albida (Del.) A. Chev. & 48,33 & 0 \\
\hline Guiera senegalensis J.F. Gmel. & 36,66 & 6,66 \\
\hline Hyphaene thebaica (L.) Mart. & 15 & 0 \\
\hline Kigelia africana (Lam.) Benth. & 13,33 & 1,66 \\
\hline Lannea fruticosa (Hochst. Ex A. Rich.) Engl. & 10 & 15 \\
\hline Piliostigma reticulatum (D.C.) Hochst. & 63,33 & 1,66 \\
\hline Prosopis africana (Guill. et Perr.) Taub. & 20 & 0 \\
\hline Sclerocarya birrea. (A. Rich.) Hochst. & 10 & 3,33 \\
\hline Sterospermum kunthianum Cham. & 1,66 & 1,66 \\
\hline Tamarindus indica L. & 3,33 & 1,66 \\
\hline Ziziphus mauritiana Lam. & 16,66 & 0 \\
\hline
\end{tabular}

Espèces non ligneuses indicatrices de la fertilité de sols:-

Les espèces non ligneuses indicatrices de la fertilité de sols et de la pauvreté des sols sont présentées au niveau du Tableau 3. Certaines espèces non ligneuses comme Dactyloctenium aegyptium (L.) Willd., Commelina forkalaei Vahl. et Cassia mimosoides L. sont les plus fréquemment citées comme espèces indicatrices de fertilité de sols. Par contre Striga hermonthica (Del.) Benth., Merremia tridentata (L.) Hallier. f. sont répertoriées comme espèces indicatrices de la pauvreté de sols. 
Tableau 3:-Espèces non ligneuses indicatrices de la fertilité de sols et de la pauvreté de sols

\begin{tabular}{|c|c|c|}
\hline Noms scientifiques & $\begin{array}{l}\text { Exploitants ayant } \\
\text { considéré l'espèce comme } \\
\text { indicatrice de fertilité de } \\
\text { sols }(\%)\end{array}$ & $\begin{array}{l}\text { Exploitants ayant } \\
\text { considérée l'espèce comme } \\
\text { indicatrice de pauvreté de } \\
\text { sols }(\%)\end{array}$ \\
\hline Alysicarpus ovalifolius (Schum. Et Thonn.) J Léonard & 16,66 & 1,66 \\
\hline Andropogon gayanus Kunth & 1,66 & 3,33 \\
\hline Andropogon pseudapricus Stapf & 3,33 & 1,66 \\
\hline Aristida longiflora Schunn. et Thonn. & 1,66 & 3,33 \\
\hline Borreria radiata D.C. & 5 & 1,66 \\
\hline Bulbostylis barbata, (Rottb.) C.B.Cl. & 0 & 1,66 \\
\hline Cassia mimosoides L. & 18,33 & 11,66 \\
\hline Cenchrus biflorus Roxb. & 15 & 1,66 \\
\hline Commelina forskalaei Vahl. & 18,33 & 3,33 \\
\hline Corchorus tridens L. & 3,33 & 0 \\
\hline Cyperus alopecuroides Rottb. & 6,66 & 1,66 \\
\hline Dactylotenium aegyptium (L.) Willd. & 21,66 & 0 \\
\hline Eragrostis tremula Hochst. Ex Steud. & 30 & 30 \\
\hline Ipomoea heterotricha F. Didr. & 5 & 3,33 \\
\hline Jacquemontia tamnifolia (L.) Griseb. & 5 & 15 \\
\hline Merremia tridentata (L.) Hallier f. & 3,33 & 45 \\
\hline Mitacarpus scaber Zucc. & 1,66 & 10 \\
\hline Monechma ciliatum (Jacq.) Milne-Redh. & 1,66 & 15 \\
\hline Pennisetum pedicellatum Trin. & 1,66 & 3,33 \\
\hline Phyllanthus pentandrus Schum. \& Thonn. & 3,33 & 0 \\
\hline Pupalia lappacea (L.) Juss. & 1,66 & 1,66 \\
\hline Sida cordifolia $\mathrm{L}$ & 0 & 10 \\
\hline Sporobolus spicatus (Vahl) Kunth. & 11,66 & 0 \\
\hline Striga hermonthica (Del.) Benth. & 0 & 38,33 \\
\hline
\end{tabular}

\section{Relevés phytosociologiques:-}

Espèces ligneuses rencontrés:-

L'inventaire des espèces ligneuses dans les champs a permis de recenser dans la commune urbaine d'Aguié, 25 espèces ligneuses réparties en 14 familles (Tableau 4).

Tableau 4:-Espèces ligneuses inventoriées par famille dans les champs paysans

\begin{tabular}{|c|c|}
\hline Familles & Noms scientifiques \\
\hline \multirow[t]{2}{*}{ Anacardiaceae } & Lannea microcarpa Engl. et K. Krauze \\
\hline & Sclerocarya birrea (A. Rich.) Hachst. \\
\hline Annonaceae & Annona senegalensis Pers. \\
\hline Arecaceae & Hyphaene thebaica (L.) Mart. \\
\hline Asclepadaceae & Calotropis procera (Ait) Ait. \\
\hline Balanitaceae & Balanites aegyptiaca (L.) Del. \\
\hline Bombacaceae & Bombax costatum Pellgr. Et Vuillet \\
\hline Burseraceae & Commiphora africana (A. Rich.) Engle. \\
\hline \multirow[t]{2}{*}{ Capparaceae } & Maerua crassifolia Forsk. \\
\hline & Boscia senegalensis (Pers.) Lam. ex Poir \\
\hline \multirow[t]{4}{*}{ Caesalpinaceae } & Bauhinia rufescens Lam. \\
\hline & Piliostigma reticulatum (DC) Hachst. \\
\hline & Cassia singueana Delile. \\
\hline & Tamarindus indica $\mathrm{L}$. \\
\hline \multirow[t]{3}{*}{ Combretaceae } & Combretum glutinosum Perr. \\
\hline & Guiera senegalensis J.F. Gmel. \\
\hline & Combretum micranthum G. Don. \\
\hline
\end{tabular}




\begin{tabular}{|l|l|}
\hline Meliaceae & Azadirachta indica A. Juss. \\
\hline \multirow{5}{*}{ Mimosaceae } & Albizia chevalerie Harms. \\
\cline { 2 - 2 } & Entada africana Guill. et Perm. \\
\cline { 2 - 2 } & Faidherbia albida Del. Chev. \\
\cline { 2 - 2 } & Prosopis africana (Guill et Perr) Lourb. \\
\hline \multirow{2}{*}{ Rhamnaceae } & Ziziphus mauritiana Lam. \\
\cline { 2 - 2 } & Ziziphus spina christi Lam. \\
\hline Verbenaceae & Vitex doniana Sweet. \\
\hline
\end{tabular}

\section{Espèces non ligneuses rencontrées:-}

Au total 35 espèces non ligneuses appartenant à 17 familles ont été répertoriées (Figure 1).

La famille des Poaceae est la plus représentée avec 22\% des espèces, suivit de la famille des Fabaceae avec $14 \%$.

Les familles des Amaranthaceae, Caesalpinaceae, Commlinaceae, Malvaceae, Pedaliaceae, Polygalaceae, Scorphulariaceae, Tiliaceae, Vitaceae, et Acanthaceae sont les moins représentées avec $2 \%$ chacune.

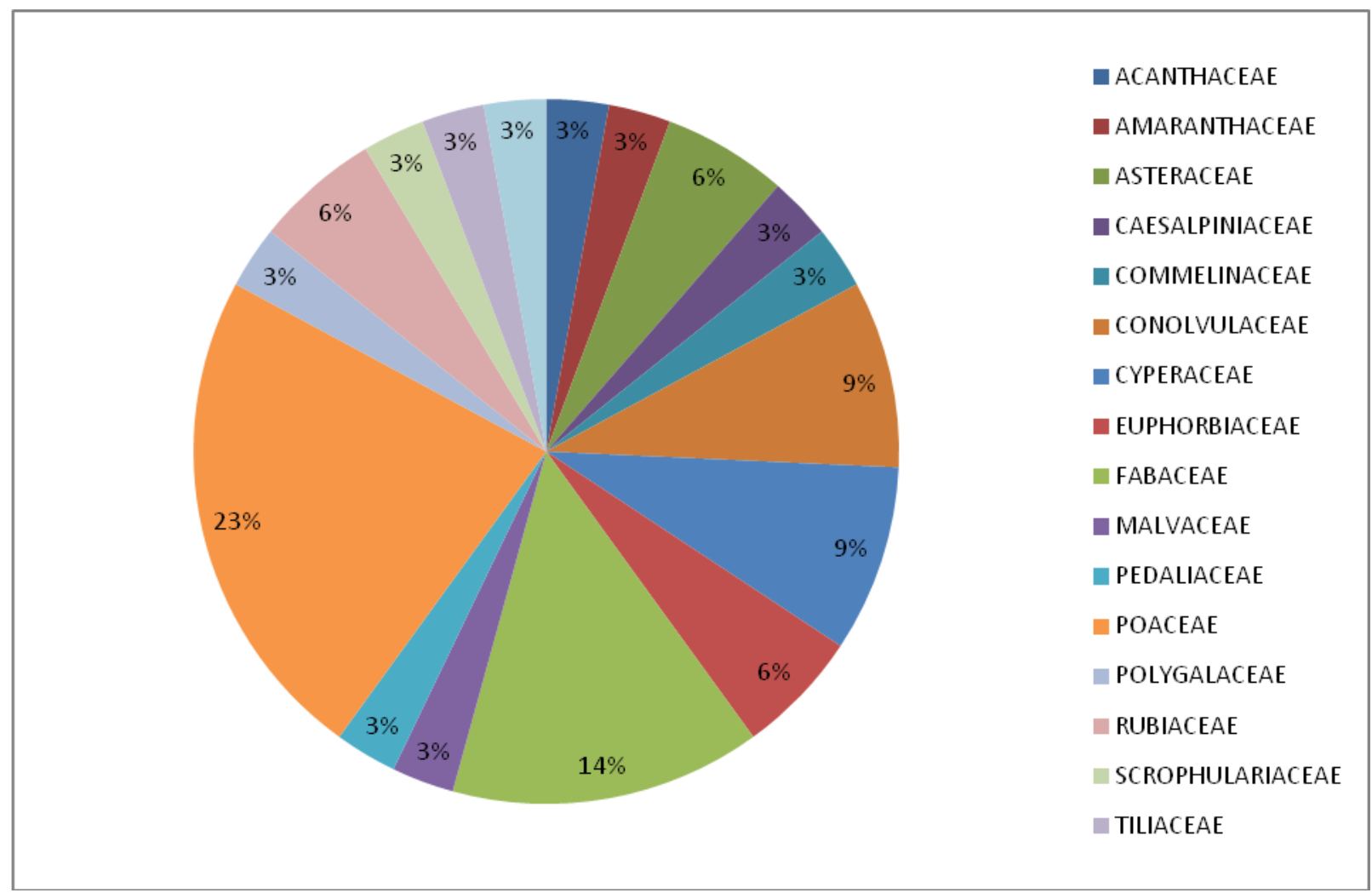

Figure 1:-Familles et proportion d'espèces herbacées rencontrées dans la commune d'Aguié

Fréquences spécifiques et contributions spécifiques des spèces non ligneuses répertoriés:-

Les fréquences spécifiques des espèces non ligneuses ainsi que leurs contributions spécifiques sont présentées au niveau du Tableau 5. Les espèces Cassia mimosoides L., Corchorus tridens L., Eragrostis tremula Hochst. Ex Steud. et Corchorus tridens ayant dans les champs à fertilités élevées des fréquences spécifiques supérieures à celles des champs à faibles fertilités sont considérées comme indicatrices de fertilité de sols. Par contre, Eragrostis tremula est une espèce indicatrice de la pauvreté de sols. 
Tableau 5:-Fréquences et contributions spécifiques des espèces non ligneuses rencontrés dans les champs à Aguié

\begin{tabular}{|c|c|c|c|c|}
\hline \multirow[t]{2}{*}{ Espèces } & \multicolumn{2}{|c|}{$\begin{array}{l}\text { Champs à fertilité } \\
\text { élevée }\end{array}$} & \multicolumn{2}{|c|}{$\begin{array}{l}\text { Champs à faible } \\
\text { fertilité }\end{array}$} \\
\hline & FSMC & CSMC & FSMC & CSMC \\
\hline Alysicarpus ovalifolius (Schum. Et Thonn.) J Léonard & 1,22 & 1,44 & 1,56 & 3,60 \\
\hline Ampelocissus africana (Lour,) & 0,13 & 0,14 & 0,13 & 0,14 \\
\hline Andropogon gayanus Kunth. & 0 & 0 & 0,54 & 0,64 \\
\hline Arachis hypogaea $\mathrm{L}$. & 2,64 & 4,52 & 0 & 0 \\
\hline Borreria scabra (Schum. Et Thonn.) K. & 3,32 & 4,64 & 2,03 & 4,82 \\
\hline Bulbostylis barbata (Rottb.) C.B.Cl. & 0 & 0 & 1,08 & 1,16 \\
\hline Cassia mimosoides $\mathrm{L}$. & 15,96 & 21,29 & 4,82 & 6,38 \\
\hline Celosia trigyna $\mathrm{L}$. & 0,81 & 0,91 & 0,13 & 0,22 \\
\hline Cenchrus biflorus Roxb. & 0 & 0 & 0,47 & 0,56 \\
\hline Chrozophora brocchiana $\mathrm{Vis}$ & 0,27 & 0,28 & 0,27 & 0,29 \\
\hline Commelina forskalaei Vahl. & 0 & 0 & 1,63 & 1,94 \\
\hline Corchorus tridens $\mathrm{L}$. & 5,16 & 6,29 & 2,71 & 3,34 \\
\hline Cyperus alopecuroides Rottb. & 3,26 & 3,47 & 0 & 0 \\
\hline Cyperus rotondis $\mathrm{L}$. & 0 & 0 & 0,40 & 0,68 \\
\hline Dactylotenium aegyptium (L.) Willd. & 0,40 & 0,43 & 0,67 & 0,72 \\
\hline Dicoma tomentosa Cass. & 0 & 0 & 0,27 & 0,29 \\
\hline Eragrostis tremula Hochst. ex Steud. & 14,67 & 18,76 & 30,63 & 45,35 \\
\hline Indigofera astragalina $\mathrm{DC}$. & 0,54 & 0,63 & 1,49 & 2,00 \\
\hline Jacquemontia tamnifolia (L.) Griseb. & 1,29 & 1,63 & 0,27 & 1,02 \\
\hline Merremia pinnata (Hochst. Ex Choisy) Hallier f. & 0 & 0 & 0,40 & 0,43 \\
\hline Merremia tridentata $(\mathrm{L}$.$) Hallier \mathrm{f}$. & 3,19 & 4,10 & 0,40 & 1,53 \\
\hline Mitacarpus scaber Zucc. & 2,03 & 2,55 & 2,58 & 3,54 \\
\hline Monechma ciliatum (Jacq.) Milne-Redh. & 1,01 & 1,20 & 3,12 & 3,63 \\
\hline Pennisetum pedicellatum Trin. & 0,33 & 0,40 & 0 & 0 \\
\hline Pennisetum typhoides (Burm) Stapf Hubb. & 5,97 & 8,06 & 0,74 & 1,76 \\
\hline Phyllanthus pentandrus Schum. \& Thonn. & 3,19 & 4,10 & 3,80 & 5,76 \\
\hline Polygala arenaria Willd. & 0 & 0 & 0,13 & 0,51 \\
\hline Sesamum alatum $\mathrm{L}$. & 0 & 0 & 0,27 & 0,45 \\
\hline Sida cordifolia $\mathrm{L}$. & 0 & 0 & 0,54 & 0,64 \\
\hline Sorghum bicolor. (L.) Moench. & 1,35 & 2,05 & 0,54 & 1,76 \\
\hline Striga hermonthica (Del.) Benth. & 0,54 & 0,64 & 1,76 & 1,89 \\
\hline Tragus racemosus (L.) All. & 0 & 0 & 0,27 & 0,32 \\
\hline Vernonia pauciflora (Willd.) Less. & 0 & 0 & 0,67 & 0,72 \\
\hline Vigna unguiculata (L.). Walp. & 10,25 & 11,79 & 1,22 & 3,74 \\
\hline Vigna subterranea L. & 0,27 & 0,56 & 0 & 0 \\
\hline
\end{tabular}

\section{Discussion:-}

\section{Espèces indicatrices de bonne fertilité de sols:-}

De nombreux systèmes agro-sylvo-pastoraux traditionnels ont montré une influence bénéfique de Faidherbia albida sur le microclimat, la fertilité des sols, et partout sur le fonctionnement des cultures associées (mil, sorgho, arachide, maïs...) (Raison, 1988). Selon Sambo (2004), Faidherbia albida est une espèce surtout utilisée pour le fourrage, mais aussi pour son rôle dans la restauration de la fertilité du sol. Sur le terroir de Yasso au Burkina Faso, les paysans considèrent Piliostigma reticulatum comme espèce indicatrice de fertilité au même titre que Dichrostachys cinerea (Sanou 2005). Dans cette étude, les espèces ligneuses indicatrices de la fertilité de sols sont Piliostigma reticulatum, Faidherbia albida, Guiera senegalensis, Dichrostachys cinerea, Bauhinia rufescens, Prosopis africana, et Ziziphus mauritiana. Ces résultats sont en conformités avec ceux de Mastalabi (2011) dans le département d'Aguié. Selon lui les sols fertiles se caractérisent par la présence de Faidherbia albida, Piliostigma reticulatum et Combretum glutinosum. Louppe en 1989 a également montré que Dichrostachys cinerea améliore les sols, car il fixe l'azote de l'air. Selon Dembelé et Ouedraogo, 1996, Guiera senegalensis et Piliostigma reticulatum, pourraient être une alternative biologique dans la conservation des eaux et des sols, pour une gestion durable des terres agricoles. 
Dans le règne végétal, les espèces non ligneuses sont les plus utilisés dans l'indication de la fertilité ou de l'infertilité des sols. Dans cette étude, les espèces non ligneuses indicatrices de la fertilité de sols sont Dactyloctenium aegyptium, Commelina forkalaei, Cassia mimosoides, Sporobolus spicatus et Cenchrus biflorus. Ces résultats sont en contradiction avec ceux de Soumana (1992), qui a montré que les paysans nigériens des régions de Say et Ouallam reconnaissent qu'une jachère est redevenue fertile grâce à des indicateurs biologiques familiers de leur environnement. Parmi les espèces végétales, les graminées comme Andropogon gayanus, Aristida mutabilis, Chloris pilosa jouent un grand rôle dans ce diagnostic. Selon Mastalabi (2011), les espèces non ligneuses indicatrices de la fertilité du sol sont: Cenchrus biflorus et Commelina benghalensis. Les espèces identifiées dans cette étude comme indicatrices de fertilité des sols diffèrent de celles communiquées par Soumana (1992) dans les régions de Say et Ouallam et de celles indiquées par Mastalabi (2011) dans le département d'Aguié. Cela peut vouloir dire que l'indication de fertilité des espèces dépend de la région dans laquelle on se trouve. Ceci s'explique par l'adaptation des espèces aux conditions du milieu. Les résultats de Mathurin (2007), ayant travaillé sur le savoir paysan de fertilité des terres au Nord-Cameroun renforce cette affirmation. En effet sur quatre terroirs de la même région, les espèces indicatrices de la fertilité du sol sont relativement différentes en fonction du terroir.

\section{Espèces indicatrices de pauvreté de sols:-}

Dans cette étude, les espèces non ligneuses indicatrices de la pauvreté de sols sont : Striga hermonthica, Merremia tridentata et Eragrostis tremula. Ces résultats sont en contradiction avec ceux de Mastalabi (2011) dans le département d'Aguié. Selon lui les sols pauvres se caractérisent par la présence de Lannea microcarpa. Mathurin (2007) au Nord-Cameroun avait trouvé les espèces suivantes comme indicatrices de la pauvreté du sol : Commelina benghalensis, Striga hermonthica, Commelina forskalaei, Ipomoea eriocarpa, Bulbostylis barbata et Digitaria horizontalis.

\section{Conclusion:-}

Il ressort de cette étude que Piliostigma reticulatum, Faidherbia albida, Guiera senegalensis, Dichrostachys cinerea, Bauhinia rufescens, Prosopis africana, et Ziziphus mauritiana sont des espèces ligneuses indicatrices de la fertilité de sols. Dactyloctenium aegyptium, Commelina forkalaei, Cassia mimosoides, Sporobolus spicatus, Cenchrus biflorus et Corchorus tridens sont des espèces non ligneuses indiquant les sols riches. Par ailleurs les espèces végétales ligneuses (Acacia nilotica, Albizia chevalieri) et les espèces non ligneuses (Striga hermonthica, Merremia tridentata, Eragrostis tremula) sont des espèces indicatrice de la pauvreté de sols.

A la lumière de nos analyses, il convient de remarquer que les sols de la zone de Dan Tchiro sont plus productifs, ensuite viennent respectivement Doramaoua, Kodaou et Dan Kada.

La fertilité étant un concept dépendant des types de culture et des conditions climatiques, une analyse physicochimiques des sols serait nécessaire pour rendre crédibles les résultats de cette étude.

\section{Remerciements:-}

Nous exprimons notre profonde gratitude au projet PASADEM, dont le financement a été très significatif pour la réalisation de ce travail. 


\section{Références:-}

1. Ali, M. (1997): Structure, fonctionnement et dynamique des parcs agroforestiers dans l'ouestdu Niger, thèse présentée pour l'obtention du grade de docteur du troisième cycle, option Biologie et Ecologie végétale, Université de Ouagadougou, Faculté des Sciences et Techniques, Laboratoire de Botanique, Biologie et Ecologie végétale, $214 \mathrm{p}$.

2. Bationo, A., Christianson, C.B., and Claij, M.C. (1996): Long term fertilizer use in Sub-Sahara Africa. Proceedings of the International Training Program on Plant Nutrient Management of Sustainable Agriculture, October 14-19, 1996, Nairobi, Kenya.

3. CTIFL. (2012): Centre technique au service de la filière fruit et légume. Le point sur lafertilité du sol en production fruitière, $\mathrm{n}^{0} 33,9 \mathrm{p}$.

4. Christel, D. (2006) : La fertilisation du sol en agrobiologie, Biobourgogne SEDARB, BPREA-CFPPA Château Chinon, 37p.

5. Dembelé, C., Ouedraogo, S.J. (1996): Potentialités de Guiera senegalensis J. F. Gmel., et Piliostigma reticulatum (DC.) Hochst. pour la conservation des eaux et des sols dans le plateau central burkinabe, INERA / Département Productions Forestières, 9p.

6. FAO (2001): Le rôle de l'agriculture dans le développement des pays les moins avancés et leur intégration à l'économiquemondiale, document préparé en vue de la Troisième Conférence des Nations Unies sur les pays les moins avancés, Bruxelles, 22p.

7. INS. (2013) : Institut National de la Statistique. Annuaire Statistique 2008 - 2012. Edition 2013. 233p.

8. Louppe, D. (1989): Production en biomasse d'un recru d'un an de Guiera senegalensis sur les sols sableux dégradés du centre nord du bassin arachidier sénégalais (village de khalyes),

9. Institut Sénégalais de Recherches Agricoles/ Direction des Recherches sur les Productions Forestières, route des pères maristes, $7 \mathrm{p}$.

10. Madstalabi, A.A. (2011): Influence de Guiera senegalensis sur la fertilité des sols et la production du mil dans la zone d'intervention du PPILDA : cas du terroir de Guidan Bakoye, rapport de stage pour l'obtention du diplôme de maitrise és science agronomiques, Facultéd'Agronomie, UAM, 62p.

11. Mathurin, M., Bassala, J.P., (2007): Savoir paysan et fertilité des terres au Nord-Cameroun, Cahiers Agricultures vol. 16, $\mathrm{n}^{\circ}$ 3, doi: 10.1684/agr.2007.0100, 185 p.Raison, J.P. (1988): Faidherbia albida (Del.) A. Chev., Revue Bois et Forets des Tropiques n $222,1989,68 \mathrm{p}$.

12. Sambo, M.B. (2004): Pratiques pastorales et biodiversité des parcours dans le canton de Dantchandou (Fakara), mémoire pour l'obtention du diplôme d'étude supérieure spécialisée,U.A.M., Faculté d'agronomie, 59p.

13. Sanou, S. (2005): Piliostigma reticulatum (D.C.) Hoscht: Potentialités fourragères et essai d'amélioration des valeurs nutritives des gousses, mémoire de fin d'étude présentée en vue de l'obtention du diplôme d'ingénieur du développement rural, Université Polytechnique de Bobo Dioulasso, département production forestière, 57p.

14. Soumana, I. (1992): «Comment les paysans différencient- ils les sols fertiles des sols Pauvres au Niger ?» Séminaire sur les recherche du sylvo-pastoralisme au Sahel, Dakar, du7 au 12 mai, 1992, Projet RCS Dakar, UNESCO, MAB- Paris.

15. Weller, S. (2013): Les principes de la fertilité des sols, construire sa relation avec le sol. Institut de recherche en agriculture biologique, FIBL, Bio Austria, Bioland Beratung, GmBh, Bio Suisse, IBLA, Luxemburg. 32p. 